Meta

Journal des traducteurs

Translators' Journal

\title{
De la traduction publicitaire à la communication multilingue
}

\section{Mathieu Guidère}

Volume 54, numéro 3, septembre 2009

URI : https://id.erudit.org/iderudit/038306ar

DOI : https://doi.org/10.7202/038306ar

Aller au sommaire du numéro

\section{Éditeur(s)}

Les Presses de l'Université de Montréal

ISSN

0026-0452 (imprimé)

1492-1421 (numérique)

Découvrir la revue

Citer cet article

Guidère, M. (2009). De la traduction publicitaire à la communication multilingue. Meta, 54(3), 417-430. https://doi.org/10.7202/038306ar

\section{Résumé de l'article}

Avec la mondialisation de l'économie, la communication commerciale occupe désormais une place prépondérante dans la vie sociale un peu partout dans le monde. La généralisation de l'Internet n'a fait que conforter cette situation en transformant la Toile mondiale en une immense vitrine commerciale. Avec ce mouvement de mondialisation, le traducteur a été de plus en plus sollicité pour aider à la diffusion des messages promotionnels à grande échelle et la traduction publicitaire a connu une progression exponentielle au cours des dernières décennies. Dans les pages qui suivent, nous allons tenter de retracer l'évolution qui a eu lieu tant sur le plan théorique que pratique. Mais l'objectif premier de l'article est d'expliquer le changement de perspective qui s'est opéré dans le domaine traductologique avec l'essor de l'Internet et le développement de la communication multilingue. Il vise également à montrer que ce changement de perspective requiert une méthode d'analyse et de travail différente pour le traducteur, dont on expose brièvement les tenants et les aboutissants. 


\title{
De la traduction publicitaire à la communication multilingue
}

\author{
MATHIEU GUIDÈRE \\ Université de Genève, Genève, Suisse \\ mathieu.guidere@unige.ch
}

\begin{abstract}
RÉSUMÉ
Avec la mondialisation de l'économie, la communication commerciale occupe désormais une place prépondérante dans la vie sociale un peu partout dans le monde. La généralisation de l'Internet n'a fait que conforter cette situation en transformant la Toile mondiale en une immense vitrine commerciale. Avec ce mouvement de mondialisation, le traducteur a été de plus en plus sollicité pour aider à la diffusion des messages promotionnels à grande échelle et la traduction publicitaire a connu une progression exponentielle au cours des dernières décennies. Dans les pages qui suivent, nous allons tenter de retracer l'évolution qui a eu lieu tant sur le plan théorique que pratique. Mais l'objectif premier de l'article est d'expliquer le changement de perspective qui s'est opéré dans le domaine traductologique avec l'essor de l'Internet et le développement de la communication multilingue. Il vise également à montrer que ce changement de perspective requiert une méthode d'analyse et de travail différente pour le traducteur, dont on expose brièvement les tenants et les aboutissants.
\end{abstract}

\begin{abstract}
With the globalization of the economy, commercial communication has become a focal point in social life all over the world. The generalization of the Internet has only confirmed this point by turning the World Wide Web into a huge commercial showcase. With this trend of globalization, the translator has been increasingly asked to help disseminate promotional messages on a large scale and advertising translation has grown exponentially over the past decades. In the following pages, we will try to highlight the evolution that has taken place both in theory and practice. But the primary objective of the article is to explain the change of perspective that came with the rise of the Internet and the development of multilingual communication in the field of Translation Studies. It also aims to show that this change in perspective requires a different method of analysis and work, which he outlines as the ins and outs for the translator.
\end{abstract}

\section{MOTS CLÉS / KEYWORDS}

publicité, adaptation, communication, publicité multilingue advertisement, adaptation, communication, multilingual advertising

\section{Réfléchir sur la traduction publicitaire}

En raison de ses difficultés intrinsèques, le texte publicitaire a très tôt intéressé les traducteurs et les traductologues de tous bords. Leur intérêt remonte aux années 1970, bien avant les linguistes qui ne se consacreront au langage et au discours publicitaires qu'au cours de la décennie suivante (Greven 1982; Tremblay 1982; EveraertDesmet 1984). Le numéro spécial de la revue Meta publié en 1972 sous la houlette de Roger Boivineau marque le début véritable de la réflexion sur la traduction publicitaire. Les contributions qui y sont réunies illustrent les préoccupations pratiques de 
l'époque, fortement marquées par les spécificités du contexte canadien en général et québécois en particulier (Paré 1972; Léonard et Siguret 1972). Mais des parallèles intéressants sont esquissés avec d'autres pays présentant une situation linguistique comparable, afin de montrer les points communs et surtout les divergences face à la pression indéniable de l'anglais (Boivineau 1972). De l'ensemble des contributions, un consensus semble néanmoins se dégager en faveur de la notion d' «adaptation" que Boivineau distingue clairement du procédé du même nom de Vinay et Darbelnet; il la définit ainsi :

L’adaptation consistera ainsi à écrire sur la trame suggérée par l'annonce originale un nouveau texte répondant aux exigences que nous avons citées. Là, il ne sera pas question de respecter scrupuleusement la pensée de l'auteur, ni même son style. Il s'agira plutôt d'atteindre le but recherché avec l'annonce originale, et la voie pour rejoindre ce but pourra s'écarter sensiblement de celle suivie par le concepteur. L'adaptateur pourra donc présenter sans rougir une de ces belles infidèles tant décriées dans d'autres domaines. Ainsi libéré, il aura la partie facile, pensera-t-on. Pas tellement car, plus qu'une belle infidèle, son adaptation devra être une belle efficace (Boivineau 1972: 15).

Cette recommandation sera suivie à la lettre par toute une génération de traducteurs francophones qui vont s'évertuer à libérer le texte publicitaire cible (c.-à-d. français) de l'emprise du texte source (c.-à-d. anglais) en élaborant toute une méthodologie de rédaction et de révision qui constitue encore aujourd'hui le socle des compétences recherchées par les agences spécialisées.

À part quelques rares comptes rendus d'expérience (Émond 1976), la réflexion sur la traduction publicitaire ne sera véritablement reprise et approfondie qu'au cours des années 1990, toujours avec la situation québécoise en ligne de mire, mais avec le mouvement de la mondialisation en arrière-plan. C'est Claude Tatilon qui reprend le premier la question posée, vingt ans plus tôt, par Roger Boivineau: «Le texte publicitaire: traduction ou adaptation?» Son article est une tentative de définition fonctionnelle du texte publicitaire pour parvenir à une méthode de traduction convaincante, c'est-à-dire permettant de concilier la fidélité et la liberté. Sa conclusion est claire:

La consigne traductologique qui me semble s'imposer est de traduire non la lettre, mais l'esprit, non les mots, mais les fonctions [en italique dans le texte]. Traduction relâchée donc, réduite à l'essentiel - l'équivalence fonctionnelle - et qu'on a sans doute intérêt à désigner d'un autre nom, adaptation me paraissant convenir tout à fait (Tatilon $1990: 245)$.

On en revient ainsi aux questions centrales de la traductologie: sens, équivalence, traduction de la lettre, traduction de l'esprit. Au cours des années 1990, les réponses à cet égard divergent en fonction du cadre théorique envisagé. Plusieurs mises au point sont publiées concernant la notion même d'adaptation, remettant en cause l'approche fonctionnelle promue par Tatilon (1990). Parallèlement, les études sur le discours publicitaire montrent la place centrale qu'occupe la rhétorique dans l'économie générale de l'annonce et la nécessité d'une approche sémiotique qui prenne en compte la relation du texte et de l'image. Adam et Bonhomme (1997) proposent une étude approfondie de la publicité écrite, dans ses composantes verbale et iconique, qui reflète bien la complexité de la communication publicitaire.

C'est qu'entre-temps, les sciences de l'information et de la communication avaient occupé le devant de la scène, reléguant la linguistique à proprement parler au second plan (Bonhomme et Rinn 1997). 
Aussi, Hatim et Mason (1997) proposent la première étude traductologique ancrée dans ce modèle théorique, mais ils passent totalement sous silence le discours publicitaire qui représente pourtant l'illustration la plus évidente de leur approche communicationnelle.

La fin des années 1990 est marquée par la montée en puissance des approches culturalistes de la traduction et de la communication (Snell-Hornby 1995; Quillard 1999; Au 1999). Concernant la publicité, plusieurs études sont consacrées à la problématique culturelle pour expliquer les difficultés de communication de plus en plus complexes qu'éprouvent les entreprises multinationales à travers le monde (Mooij 1998). Katan (1999) propose une sorte de manuel destiné aux traducteurs présentés alors comme des «négociateurs» et des «médiateurs» entre les cultures, mais il ne traite pas spécifiquement de la publicité.

Il faut attendre le début des années 2000 pour voir paraître la première étude d'ensemble exclusivement consacrée à la traduction publicitaire. Guidère (2000) s'appuie sur un corpus homogène d'annonces multilingues qui lui permet d'aborder les principales difficultés soulevées par la «traduction» des publicités françaises vers l'anglais, l'arabe et l'espagnol. À travers une série d'études de cas concrets, il propose une description exhaustive des différents niveaux d'analyse en expliquant, à chaque fois, les choix de traduction et les décisions prises par le traducteur.

Cette réflexion sera poursuivie au cours des années suivantes, toujours à partir de cas concrets pour tenter de rendre compte des évolutions du marché en matière de traductions publicitaires (Guidère 2004). Elle sera couronnée par une étude sur «la communication multilingue» qui représente, pour l'auteur, le nouveau cadre d'intervention du traducteur à l'ère de la mondialisation et de l'Internet triomphant (Guidère 2008).

Entre-temps, on assiste à un véritable regain d'intérêt pour les problématiques propres à la traduction publicitaire. Künzli (2001) s'intéresse à la traduction du dialogue dans les textes publicitaires et à la différence constatée entre traducteurs «experts» et «novices» (Künzli 2001). Adab (2002) aborde la question de la qualité et de l'évaluation, avant de revenir sur les principaux débats qui agitent les spécialistes de traduction publicitaire (Adab et Rodriguez 2004). Smith (2006) s'intéresse à la traduction des slogans d'un point de vue rhétorique; Baider et Lamprou (2007) se penchent sur la traduction du non-dit d'un point de vue cognitif. Enfin, les aires culturelles concernées par les études traductologiques s'élargissent et se diversifient (Sidiropoulou 2008; Quillard 2006), mais la Chine occupe désormais une place de plus en plus importante en raison des problèmes posés ces dernières années par certaines campagnes occidentales et des difficultés réelles éprouvées lors de leur adaptation publicitaire (O’Barr 2007; Fengru et Shooshtari 2007).

\section{2. Évolution de la traduction publicitaire}

Dans la pratique professionnelle, les traducteurs ont assisté à une évolution rapide aussi bien sur le plan des missions qui leur sont confiées que des méthodes de travail employées pour les accomplir.

Ainsi, il est possible de constater qu'au commencement de la traduction publicitaire, il y avait surtout «le verbe», c'est-à-dire le seul texte publicitaire qui nécessitait 
un traitement spécifique. La principale mission des traducteurs se limitait à transférer un message promotionnel d'une langue à l'autre.

Dans le transfert, la traduction avait pour objet principal et quasi exclusif le texte publicitaire. Par texte publicitaire, il faut entendre les différentes parties rédigées du message promotionnel, à savoir: l'appellation (nom de la marque et/ou du produit), le slogan (phrase d'accroche et/ou phrase d'assise), et le rédactionnel (texte d'explication et/ou argumentaire commercial). Les traits caractéristiques de cette phase de la pratique professionnelle peuvent être assimilés à des erreurs de débutants en traduction puisqu'on peut les résumer en deux mots: souci du sens littéral et prise en compte partielle du contexte. En d'autres termes, les premiers traducteurs de textes publicitaires avaient pris - consciemment ou inconsciemment - le critère de fidélité à l'original comme principal repère pour la transposition des messages. Ainsi, la traduction fidèle à la source apparaît comme le fait remarquable de cette première phase d'exercice professionnel.

Mais très vite, les traducteurs se sont rendu compte de l'inadéquation du critère de fidélité pour ce genre de textes, dont le contenu et le but n'avaient rien de sacral pour exiger un respect scrupuleux de la forme ni même du fond. De ce constat de décalage entre la visée du message et le type d'équivalence recherchée naît la consigne d'adaptation pour les textes publicitaires.

L'adaptation marque un tournant salvateur dans l'exercice professionnel de la traduction publicitaire, mais elle a donné lieu à une multitude de pratiques qui oscillent entre la reformulation métaphorique et la réécriture totale du texte original. Grâce à l'adaptation, la pratique de la traduction publicitaire entre dans une nouvelle phase où les professionnels vont rechercher une «équivalence fonctionnelle» (Tatilon 1990 : 245) des messages publicitaires. Autrement dit, le traducteur se pose désormais la question de la finalité pragmatique du message et pense sa traduction en fonction d'un but précis, généralement un effet à produire sur le récepteur à partir d'un argumentaire particulier.

Sur le plan professionnel, l'intérêt des traducteurs s'est ainsi déplacé de la «source» vers la «cible», avec une valorisation de la praxis langagière par rapport à la forme et au contenu textuel. Ce qui compte désormais dans le message publicitaire, ce n'est pas tant ce qu'il dit, mais ce qu'il vise à faire faire (promouvoir, apprécier, acheter le produit). Ce changement de perspective a été la principale innovation méthodologique durant les années 1990 où l'on voit se développer une forme de traduction publicitaire de nature cibliste, c'est-à-dire qui essaie de tenir compte des contraintes socio-économiques du genre, notamment concernant la réception des signes verbaux et des signes iconiques. Ainsi, à travers les exemples de cette période, on voit se dessiner une nouvelle étape dans la pratique de la traduction publicitaire: il s'agit de la prise en compte de l'image dans le processus d'adaptation mené par les traducteurs (Guidère 2000: 217-245).

En effet, progressivement, les traducteurs vont avoir accès aux images accompagnant les textes qu'ils doivent traduire et vont en tirer profit pour adapter leurs traductions en tenant compte de l'iconographie qui accompagne le rédactionnel. Il y a eu ainsi une conscience réelle du poids des icônes et une volonté affichée de travailler dans le sens d'une complémentarité effective entre le texte et l'image pour assurer une meilleure efficience des messages publicitaires. À ce stade de la pratique, on peut dire que la traduction se faisait en fonction de l'image, mais le travail du traducteur 
ne portait pas encore précisément sur la modification technique de l'iconographie publicitaire.

La pratique actuelle peut être datée du début des années 2000, au tournant du siècle. À ce moment-là, profitant des possibilités offertes par les technologies de l'information et de la communication, certains traducteurs ont eu l'ingénieuse idée de proposer une version adaptée en même temps sur le plan du texte et de l'image, celleci étant quelque peu retouchée à des fins d'illustration et de persuasion du commanditaire. Ils voulaient par là suggérer des changements iconographiques qui leur semblaient utiles pour assurer un meilleur accueil du message publicitaire dans les pays cibles. En plus de la traduction, ils faisaient œuvre de médiation interculturelle.

Mais de telles adaptations iconographiques n'auraient pas pu être possibles sans les progrès techniques réalisés en matière de logiciels bureautiques et sans la maîtrise sans cesse grandissante des outils informatiques de la part des traducteurs, en particulier des logiciels d'aide à la traduction, d'infographie et de retouche des images.

Toujours est-il que les suggestions faites par les traducteurs ont été appréciées et validées par les spécialistes de la communication au sein des agences spécialisées et des entreprises multinationales, lesquelles découvraient ainsi que les traducteurs possédaient des compétences autres que la simple maîtrise des langues. Ils étaient non seulement dépositaires d'un savoir culturel vendeur mais également détenteurs d'un savoir-faire technique susceptible de faciliter la recontextualisation du message en réduisant le nombre des intervenants lors de l'adaptation, c'est-à-dire en termes économiques, les délais de fabrication et les coûts de production. De cette concentration des compétences est née une nouvelle spécialité ayant pour nom la localisation publicitaire, qui représente depuis quelques années la phase ultime de cette évolution de la profession.

La localisation publicitaire consiste à adapter une communication commerciale à un locus (province, pays, région, continent) en prenant en charge la totalité du processus d'adaptation textuelle et iconographique. Cela signifie que le traducteur est maître d'œuvre pour l'intégralité du message et qu'il est responsable à la fois de la traduction du texte, des retouches éventuelles des images qui l'accompagnent, mais également de la mise en forme finale de la communication publicitaire: ajustements éventuels du texte et de l'image, choix des couleurs, adaptation des symboles, etc.

Dans le détail, cette évolution représente un saut qualitatif important qui a permis de briser le carcan de la pratique professionnelle jusque-là cantonnée dans le seul domaine linguistique et textuel. La «localisation» apparaît alors comme la solution d'une libération tant attendue. Non seulement elle permet d'élargir le champ d'intervention des traducteurs - et par là le marché de la traduction -, mais elle ouvre également des perspectives d'exercice et de carrière, voire de rémunération, parfois inattendues pour ce traducteur localisateur qui possède des compétences techniques et culturelles élargies.

En effet, le marché de la localisation fait appel à différents outils qui relèvent aussi bien de la traduction assistée par ordinateur (TAO) que de l'ingénierie linguistique et de l'informatique: mémoires de traduction, outils de localisation logicielle et de recherche terminologique, logiciels de mise en page, de maquette et d'infographie, etc. Tous ces outils sont conçus pour aider le traducteur dans ses nouvelles tâches de communicateur. 


\section{Traduction publicitaire et communication multilingue}

L'idée que la traduction s'apparente à un acte de communication qui a lieu en deux ou plusieurs langues apparaît au début des années 1990. Dans leur ouvrage Discourse and the Translator, Hatim et Mason (1990) affichaient déjà un objectif clair pour leur étude: "L'objet central de ce livre est la traduction envisagée comme un processus de communication qui a lieu à l'intérieur d'un contexte social» (Hatim et Mason 1990: 20). Cette optique sera élargie et développée sept ans plus tard grâce à Translator as Communicator (Hatim et Mason 1997). Dans cet ouvrage, les auteurs appellent à une unification de la discipline - et des traducteurs - autour de leur point commun, à savoir la communication. Pour eux, qu'ils soient traducteurs littéraires ou audiovisuels, adaptateurs ou interprètes de conférence, tous sont avant tout des communicateurs. À travers une série d'études de cas, Hatim et Mason illustrent leur propos en prenant un exemple de chaque domaine d'application: «la texture en interprétation simultanée» (chapitre 4); «la politesse en traduction audiovisuelle» (chapitre 5); «les idiolectes dans la traduction littéraire» (chapitre 6); «la forme et la fonction dans la traduction des textes sacrés et des textes sensibles" (chapitre 7); etc. Par ces exemples, les auteurs recherchent les fondements d'un modèle d'analyse des textes parce qu'ils sont convaincus que seule l'approche textuelle peut rendre compte de la traduction en tant que communication. D’ailleurs, les développements qu'ils consacrent à la dimension didactique de leur approche communicationnelle visent l'application de la linguistique textuelle à la formation des traducteurs et à l'évaluation de leur compétence.

Malgré son caractère innovant, ce modèle d'analyse est rendu caduc au cours des années 2000 avec l'essor que l'on connaît de l'Internet et de la communication multilingue sur la Toile mondiale. En effet, la communication est devenue plurielle en raison de la pluralité des récepteurs et des langues disponibles sur la Toile mondiale. La quantité sans cesse grandissante de ce nouveau type de productions multilingues incite à donner toute leur mesure aux phénomènes langagiers de la mondialisation et de l'Internet. La rareté des recherches dans ce domaine contraste avec l'abondance de matière première disponible pour l'étude et l'analyse. Pourtant, les questions ne manquent pas: la pluralité des versions adaptées d'un même texte promotionnel renvoiet-elle à une pluralité de conceptions? La différenciation des textes et des images lors de l'adaptation cache-t-elle une différence de stratégies communicationnelles? Comment se fait dans la pratique la résolution des dilemmes liés aux relations conflictuelles de l'identité et de l'altérité? Comment le traducteur assure-t-il la permanence du contenu et de l'expression par-delà la diversité linguistique et culturelle?

Si l'on ajoute à ces questions celles que pose la prise en considération des spécificités proprement communicationnelles des messages, et notamment de la dimension pragmatique du discours commercial ou institutionnel, l'on se trouve confronté à des problématiques essentielles tant du point de vue théorique que pratique: la praxis langagière s'exprime-t-elle pareillement d'une langue-culture à une autre langueculture? Comment se fait l'adaptation de cette praxis sur le plan du texte, du son et de l'image? Les effets de la version adaptée sont-ils comparables à ceux de l'original? Enfin, comment juger la qualité d'une adaptation par-delà la multiplicité des versions?

Dans La Communication multilingue (Guidère 2008), l'auteur montre l'intérêt et les enjeux de ces questions aussi bien pour une étude générale de la traduction que 
pour une approche comparative de la communication. Il explique, à partir d'études de cas et d'exemples concrets, la nécessité de dépasser l'analyse du discours pour une véritable prise en compte des formes de communication nouvelles qui se déploient aujourd'hui parallèlement et simultanément en plusieurs langues et notamment sur la Toile.

Comme la «traduction», l'auteur montre que l'expression communication multilingue est polysémique et qu'elle désigne des aspects différents selon la perspective adoptée:

1) Un message promotionnel conçu en plusieurs langues: la communication est ellemême multilingue.

2) Un message conçu en une seule langue, mais diffusé en plusieurs langues: la communication est unilingue, mais la diffusion est multilingue.

3) Un message issu d'un environnement multiculturel: la communication est multilingue du fait du contexte multilingue (par ex. deux ou plusieurs langues officielles dans un même pays).

Dans la pratique, ces distinctions se trouvent illustrées sur le plan du multitexte. Celui-ci désigne la pluralité des messages diffusés en même temps, pour une même communication, en plusieurs langues. Le facteur temporel (fT) est ce qui différencie fondamentalement le multitexte de la simple traduction : la notion de «texte source» cède la place à une pluralité de textes rédigés parallèlement et quasi simultanément. Ceux-ci sont de plus en plus multilingues et multimédias: non seulement ils intègrent dans leur création texte, son et image, mais encore ils sont véhiculés par plusieurs canaux d'information et de communication (presse, radio, télévision, Internet).

Sur le plan langagier, le message n'est pas seulement traduit en un certain nombre d'idiomes, il est également décliné en plusieurs versions en fonction du pays et du public cible. Que ce soit sur le plan national ou international, il est possible de trouver différentes mises en forme d'un même message (par ex. une publicité pour les cosmétiques) sur un même support (par ex. un magazine féminin). De ce point de vue, le multitexte renvoie aussi bien à la diversité des formulations et des aménagements que peut subir un même message au sein d'une même langue qu'entre deux langues-cultures. Les indices de l'identité, comme ceux de l'altérité, sont perceptibles tant sur le plan de l'expression que de la forme du message.

Enfin, sur le plan communicationnel, le multitexte désigne le résultat des différentes formes d'adaptation: transposition, retouche, ou encore réécriture.

Dans cette perspective, plusieurs aspects de la communication multilingue méritent d'être signalés. Tout d'abord, la production des différentes versions d'un même message - le multitexte - se fait soit de façon simultanée, soit de façon décalée, selon la stratégie d'internationalisation adoptée par l'entreprise. La transposition du message dans l'ensemble des langues-cultures visées a lieu dans des «agences d'adaptation multilingue» qui coordonnent le travail des différentes équipes spécialisées par aire géographique ou linguistique (ex. agence Ursula Grüber en France). Cette précision est importante parce qu'elle définit le multitexte par rapport à une temporalité et à une spatialité qui lui sont inhérentes. Celles-ci ne concernent pas tant les délais de réalisation des versions locales que l'exigence de concomitance dans la diffusion territoriale des différentes versions. Une telle exigence est censée garantir l'identité de la communication par-delà la diversité des langues et des cultures. 
Ensuite, le multitexte communicationnel se distingue par une dimension proprement téléologique. La pluralité des versions possède une finalité affichée aussi bien sur le plan de l'élaboration que de la diffusion. Outre le fait que la communication est discursivement orientée vers la persuasion, l'ensemble du processus est remarquable par sa visée tactique. Adapter un même message à plusieurs cultures n'a pas toujours pour objectif d'accompagner la mise sur le marché des services ou produits qu'il vante. La version adaptée de la communication peut être diffusée parfois sans même que le produit soit disponible sur le marché cible. Dans ce cas, l'adaptation doit être examinée à la lumière de l'intérêt tactique de l'adaptation. Généralement, celle-ci a pour fonction d'occuper discursivement un créneau face à une concurrence qui est commercialement en avance. La diffusion de la version adaptée crée chez les récepteurs la conviction de l'existence et de la disponibilité des produits et services indiqués. Cette manœuvre, désormais classique en marketing global, permet non seulement d'attiser l'attente des consommateurs locaux pour un nouveau produit, mais elle bloque également les initiatives des concurrents qui se trouvent pris de court par un simple effet d'annonce.

Enfin, le multitexte renvoie à un ensemble de contraintes formelles qui font la spécificité de la communication multilingue. La pluralité des versions n'est possible que s'il existe des normes strictes sur le plan technique et rédactionnel. L'identité plurielle du message est le résultat d'une codification, aussi draconienne que fonctionnelle, des modalités de l'adaptation. Cela signifie que la communication ne se réfère pas systématiquement à une langue source et à une culture d'origine; dans la pratique, elle s'effectue par le biais d'un compromis qui, dès le stade de création, intègre l'idée d'une adaptation à plusieurs langues et cultures. De la sorte, il existe bien un message original, mais il est à envisager comme un Urtext (texte premier). La mise en évidence des différentes versions permet de retrouver l'esprit de la création originale, et leur comparaison incite à réfléchir sur la communication multilingue dans son ensemble.

\section{Le traducteur publicitaire et la nouvelle tour de Babel}

Cette tendance à la multiplication des versions traduites et adaptées s'est accélérée ces dernières années avec l'extension et le succès que l'on connaît de l'Internet. Avec l'explosion du volume de messages publicitaires et du nombre de langues dans le cybermonde, l'Internet rappelle davantage la mythique tour de Babel que la simple toile d'araignée $(W e b)$. La simple navigation met l'internaute face à un nombre considérable de sites et de messages disponibles dans diverses langues du monde.

Sur le plan de la communication, l'Internet est vertigineux à plus d'un titre: d'abord, par le nombre sans cesse plus grand de personnes qui y prennent part; ensuite, par la somme sans cesse croissante de pages mises en ligne; enfin, par le nombre exponentiel de sites web disponibles en plusieurs langues. Par son multilinguisme triomphant, l'Internet forme la tour de Babel de notre temps. En effet, le multilinguisme est probablement le trait le plus marquant de l'Internet, mais les sites web disponibles en plusieurs langues offrent une telle variété de contenus et de présentations qu'il est difficile de dresser une typologie fiable et stable. Il est clair cependant que l'argument linguistique, c'est-à-dire la possibilité d'accéder au site et à l'information dans la langue maternelle de l'internaute, est de plus en plus mis en avant dans les stratégies de communication sur la Toile. 
Le site web multilingue est ainsi devenu une réalité tangible et une donnée incontournable dans les études traductologiques. Suivant l'expansion de l'entreprise et son implantation à l'étranger, son site évolue en même temps que le marketing et s'adapte aux exigences des marchés visés. Les diverses versions adaptées d'un même site donnent ainsi la mesure du travail effectué en amont par les traducteurs sur le plan de la communication. Il est possible, en effet, d'explorer cet Internet multilingue en suivant la trace des traducteurs professionnels qui s'occupent de l'adaptation des messages promotionnels sur la Toile un peu partout dans le monde. Le lien étroit avec la publicité internationale est assuré par le fait que les sites web représentent aujourd'hui une nouvelle forme de communication publicitaire, puisqu'ils permettent de faire la promotion, de façon directe ou indirecte, des produits et services affichés sur la Toile (Janoschka 2004). Ainsi pour les entreprises de tous secteurs, ces sites représentent une immense vitrine commerciale, ouverte sur le marché mondial grâce au réseau de l'Internet. Pour les institutions internationales et les organisations non gouvernementales, les sites web représentent un excellent moyen de communiquer à moindres frais avec un nombre illimité de personnes. Enfin, pour le simple citoyen, le blogue ou le site personnel est devenu le moyen de communication par excellence avec le reste des internautes, lui permettant à la fois d'exprimer ses opinions et de participer à la vie de la Cité. Tant par leur fonction que par leur conception, ces nouvelles formes de communication partagent de nombreux points communs. Ainsi, l'adaptation d'une publicité, comme celle d'un site web, nécessite la maîtrise de savoirs sémiotiques et techniques similaires. À défaut d'une réflexion d'ensemble sur la communication multilingue, le traducteur serait condamné à travailler dans l'urgence et la précarité, sans souci de durée ni de continuité. Et ce tort lui incombe autant qu'au commanditaire: à lui, parce qu'il n'aura pas tenu compte des contraintes de base de la communication multilingue; à son commanditaire pour les économies de bouts de chandelles qu'il paiera cher en termes d'image de marque.

Actuellement, la gamme des prestations offertes par les agences de traduction publicitaire est riche et variée: elle porte sur l'adaptation des argumentaires de vente, des manuels d'identité visuelle, des chartes graphiques et des styles rédactionnels, des brochures internes à l'entreprise, des communiqués de presse et de relations publiques, des dossiers de presse, des annonces et des courriers publicitaires, des campagnes de lancements de produits, des prospectus de présentation des services, des slogans de marque et de produit, des affiches publicitaires, des textes de promotion sur les sites web, des spots publicitaires pour la télévision et la radio, des emballages et des étiquettes destinés à l'export. Ces services concernent tous les secteurs d'activité. La variété des thèmes abordés dans les traductions publicitaires va des annonces de services financiers aux brochures de mode, en passant par les cartes de menus, les enquêtes d'opinion et les études de marché. La traduction d'annonces publicitaires pour de nouvelles voitures ou motos, y compris des rapports d'essais, fait également partie du champ de la communication multilingue. Les services s'étendent en outre aux activités de copywriting et d'adaptation des story-boards dans le cadre des campagnes d'affichage et de promotion commerciale à l'international. Enfin, les agences spécialisées offrent des services de veille médias et de revue de presse multilingue qui portent sur l'analyse de la concurrence dans plusieurs langues, de la communication et des campagnes de promotion dans les pays étrangers, ainsi que des services de relecture et de contrôle final de tous les produits de communication publicitaire. 
Malgré cette diversité de services et de prestations qui a constitué un bouleversement majeur du marché du travail au cours de la dernière décennie, la théorie de la traduction n'a pas suffisamment pris en considération les conséquences inévitables de ces innovations pour le métier de traducteur. Ni les théoriciens ni les praticiens n'ont suffisamment analysé le changement remarquable de perspective et de méthode qui s'est opéré dans le domaine langagier et, en premier lieu, dans celui de la communication multilingue.

Deux aspects attirent pourtant l'attention parce qu'ils induisent une nouvelle conception de la traduction et une vision renouvelée du traducteur. Le premier élément de fond est le phénomène d'instrumentalisation accrue des langues locales. Celles-ci s'affirment de plus en plus comme un outil idéologique et comme un enjeu politique de premier plan, deux aspects dont il n'est plus possible de faire abstraction dans la plupart des régions du monde, y compris dans le domaine strictement commercial (Guidère 2008: 61-68). Le second élément est celui d'une connaissance de plus en plus fine des processus cognitifs mis en œuvre par l'humain dans la réalisation d'une traduction, et cela, quelle que soit la nature du message à traduire (Vandaele et Lubin 2005). Même «l'intuition» du traducteur est de mieux en mieux connue et analysée de façon scientifique dans le cadre des sciences cognitives. L'interdisciplinarité a donc permis d'appréhender la complexité d'un métier que l'on croyait insondable.

Ces découvertes sont articulées autour du concept de «réflexivité» qui englobe à la fois la réflexion du traducteur sur son travail et le caractère réflexif du processus de traduction. Dans le cadre de la communication multilingue, le texte à traduire n'est pas simplement un assemblage de mots ou d'expressions plus ou moins marquées culturellement, il est envisagé comme une matrice cohérente et finalisée de sens connotés.

La traduction analytique qui prend corps avec l'essor de la communication multilingue sur la Toile récuse plusieurs postulats de la traductologie classique (Guidère 2008: 94-103):

1) Elle refuse la séparation du texte à traduire et du sujet traduisant, car les deux sont indissociables dans l'activité de traduction: l'un fait exister l'autre et l'actualise.

2) Elle récuse le fait que le «sens» soit dans le texte: il est un devenir tributaire de la compétence du traducteur qui l'actualise, que ce soit au départ ou à l'arrivée du processus.

3) Elle refuse le caractère énigmatique du «sens» et le considère comme une orientation de l'esprit du traducteur à un moment donné concernant un sujet particulier. Interpréter n'est pas traduire: l'interprétation elle-même est tributaire de la compréhension du traducteur.

4) Elle conteste la fonctionnalisation du texte à traduire parce que la fonction ou le skopos sont eux-mêmes des données subjectives, changeantes et dépendantes du traducteur. La finalité de l'acte de traduire elle-même est évolutive et circonstancielle; elle ne peut être prise comme fondement à la traduction.

Bref, la traduction analytique vise l'autonomisation du traducteur et s'oppose à une vision réductrice de sa fonction, parce que le traducteur fait autant exister la communication que celle-ci ne le fait exister. Lacte de traduire est l'enjeu central de la réflexion parce qu'il fait exister simultanément la communication pour le traducteur et le traducteur dans le monde. En se débarrassant de l'idée d'une primauté du 
sens dans l'acte de traduire, on peut davantage mettre en valeur l'existence de la traduction en elle-même.

Mais comment saisir le propre de la traduction dans ce nouveau contexte global?

Les travaux du philosophe Jean-Paul Sartre sur l'imagination créatrice permettent d'entrevoir une réponse à cette question.

On doit apprendre les objets, c'est-à-dire multiplier sur eux les points de vue possibles. L'objet lui-même est la synthèse de toutes ces apparitions [...] Qu'est-ce que cela signifie pour nous? La nécessité de faire le tour des objets (Sartre 1940: 22).

Cela appelle une ouverture du traducteur à un savoir qu'il n'a pas encore, c'est la nécessaire exploration de l'objet par étapes et progressivement. L'exemple du «cube» décrit par Sartre lui-même permet de saisir la complexité du phénomène d'appréhension de la traduction:

L'objet, quoi qu'il entre tout entier dans ma perception, ne m'est jamais donné que d'un côté à la fois. On connaît l'exemple du cube: je ne puis savoir que c'est un cube tant que je n'ai pas appréhendé ses six faces; je puis à la rigueur en voir trois à la fois, mais jamais plus. Il faut donc que je les appréhende successivement (Sartre 1940: 21).

Dans la communication multilingue, le message à traduire est généralement un objet présent devant moi comme objet à connaître. Certes, il peut être un texte électronique, mais cela n'enlève rien à son caractère sensible, concret, d'objet-à-traduire. Le problème n'est donc pas dans l'objet mais dans la manière de l'appréhender. C'est là que l'image $\mathrm{du}$ «cube traductologique» est utile. Nous proposons une représentation de l'objet traductionnel, c'est-à-dire du sens à traduire, suivant la conception réflexive évoquée précédemment en représentant les différentes faces du cube par une alternance de conceptions (C), de perceptions (P) et d'intentions (I):

\section{FIGURE 1}

Le cube traductologique

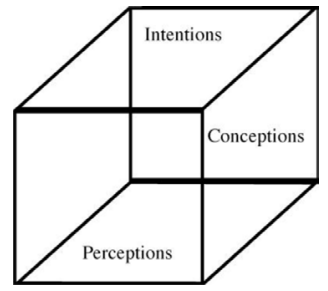

L'image du cube traductologique permet de saisir la complexité du processus de traduction dans la communication publicitaire multilingue. Le cube possède, en effet, six faces, mais il n'est possible d'en voir que trois à la fois. Les faces accessibles au traducteur sont celles de ses propres conceptions, de ses propres perceptions et de ses propres intentions. Mais il doit nécessairement retourner le cube pour voir les conceptions, les perceptions et les intentions des «Autres», ceux qui parlent à travers le texte / message source. Cette image familière et dynamique permet de comprendre que le processus de traduction correspond fondamentalement à une activité réflexive en perpétuel ajustement. C'est pourquoi elle ouvre des champs nouveaux d'exploitation 
didactique. La méthodologie de base consisterait à enseigner le processus de traduction suivant trois étapes essentielles: déconstruction du cube traductologique (c.-à-d. les facettes de l'original), choix des facettes pertinentes pour la traduction (c.-à-d. l'orientation du message), reconstruction du cube par le traducteur (c.-à-d. les facettes correspondant à la finalité et à la cible). Le cube traductologique permet de mieux comprendre le lien qui unit le monde subjectif des traducteurs aux données objectives des messages à traduire.

Dans la communication publicitaire multilingue, les interprètes-traducteurs doivent procéder à une analyse en trois temps qui représente les trois grandes phases logiques et chronologiques dans l'élaboration d'une traduction. La première phase est celle de l'analyse des concepts présents dans le message source. Elle concerne les idées développées et les notions centrales qui structurent l'objet-à-traduire. Dans cette phase, il s'agit de décortiquer la compréhension par l'esprit des mouvements du texte en référence aux acquis antérieurs du traducteur. Les mots employés dans le texte original ne correspondent pas nécessairement aux objets connus du traducteur dans sa langue maternelle, mais ils permettent par analogie le déclenchement du processus traductionnel. La conjonction des significations textuelles et mentales autorise le déchiffrement et l'interprétation du texte, mais elle doit être réalisée de façon consciente et consciencieuse. La deuxième phase est celle de l'analyse des percepts (Deleuze 1981) que déclenche le texte source. Elle concerne l'effet des mots présents dans le message à traduire sur les facultés du sujet traduisant et, au-delà, sur les récepteurs finaux du texte. Cet effet est un mouvement qui va des mots jusqu'au cerveau en passant par divers centres nerveux et mémoriels. Le mécanisme de la compréhension possède une dimension perceptive dans laquelle les éléments sémantiques du message interagissent avec les sens mémorisés par le traducteur pour aboutir à une impression particulière que celui-ci devra décider de rendre ou d'ignorer dans sa traduction. La troisième phase est celle de l'intention, c'est-à-dire du choix d'une orientation à donner au message final. Elle concerne la mise en forme de la combinaison de "percepts» et de "concepts» issue des deux phases précédentes suivant une visée particulière, qu'elle soit de nature personnelle, collective ou institutionnelle. L'activité d'équivalence à proprement parler est le moment clé de cette phase intentionnelle parce qu'elle correspond précisément à l'assignation d'un sens téléologique, général ou particulier, observable aux plans macro- ou microtextuels. Ainsi envisagée, la traduction apparaît en définitive comme une communication multilingue d'essence analytique et dynamique.

\section{RÉFÉRENCES}

AdAB, Beverly (2002): The Translation of Advertising: A Framework for Evaluation. Babel. 47(2):133-157.

Adab, Beverly et Valdés Rodriguez, Cristina (2004): Key Debates in The Translation of Advertising Material. The Translator. 10(2). Manchester: St Jerome.

Adam, Jean-Michel et Bonнomme, Marc (1997): L'argumentation publicitaire. Rhétorique de l'éloge et de la persuasion. Paris: Nathan.

Au, Kenneth Kim-Lung (1999): Cultural Transfer in Advertisement Translation. Babel. 45(2):97106.

Baider, Fabienne et Lamprou, Efi (2007): La traduction du non-traduit dans les publicités à Chypre: Quels enjeux culturels? Quels procédés cognitifs? Meta. 52(1):93-107.

Bastin, Georges (1993): La notion d'adaptation en traduction. Meta. 38(3):473-478. 
Boivineau, Roger (1972): L'abc de l'adaptation publicitaire. Meta. 17(1):5-28.

BoIvineau, Roger (1972): Que pense-t-on de l'adaptation publicitaire en Belgique et en Suisse? Meta. 17(1):47-51.

Bonhomme, Marc et Rinn, Michael (1997): Peut-on traduire la publicité? L'exemple des annonces romandes et alémaniques. In: Pascal Singy et Peter Trudgill, dir. Communication et pragmatique interculturelles. Bulletin suisse de linguistique appliquée. 65. Neuchâtel, Institut de linguistique de l'Université de Neuchâtel, 9-24.

Cathelat, Bernard (2001): Publicité et société. Paris: Payot.

Deleuze, Gilles (1981): Francis Bacon. Logique de la sensation. Paris: Seuil.

Eco, Umberto (2004): Mouse or Rat? Translation as Negotiation. London: Phoenix.

Émond, Philippe (1976): La traduction dans les agences de publicité. Meta. 21(1):81-86.

Everaert-Desmet, Nicole (1984): La communication publicitaire: étude sémio-pragmatique. Louvain-la-Neuve: Cabay.

Fuentes Luque, Adrián et Kelly, Dorothy (2000): The Translator as Mediator in Advertising Spanish Products in English-Speaking Markets. In: Allison Beeby, Doris Ensinger et Marisa Presas, dir. Investigating Translation. Amsterdam: John Benjamins, 235-242.

Gambier, Yves (1992): Adaptation: une ambiguité à interroger. Meta. 37(3):421-425.

Greven, Hubert A. (1982): La langue des slogans publicitaires en anglais contemporain. Paris: PUF.

Guidère, Mathieu (2000): Aspects de la traduction publicitaire. Babel. 46(1):20-40.

Guidère, Mathieu (2000): Publicité et traduction. Paris: L'Harmattan.

GuIDÈre, Mathieu (2001): Sémiotique comparée du territoire. Les stratégies territoriales en publicité internationale. In: Dominique Pages et Nicolas Pelissier, dir. Territoires sous influence. Paris: L'Harmattan, 121-143.

Guidère, Mathieu (2002): La traduction publicitaire et ses perspectives d'avenir. Hieronymus. $2: 9-15$

Guidère, Mathieu (2004): De l'adaptation à la localisation publicitaire. In: James ArCHIBALd, dir. La localisation: problématique de la formation. Montréal: Linguatech, 69-96.

Guidère, Mathieu (2008): Traduire la publicité ou Comment argumenter ses choix de traduction. Traduire. 219:22-46.

GUIDÈRE, Mathieu (2008): La Communication multilingue: traduction commerciale et institutionnelle. Paris: De Boeck Université. Coll. «Traducto».

Hatim, Basil et Mason, Ian (1990): Discourse and the Translator. London: Longman.

Hatim, Basil et Mason, Ian (1997): The Translator as Communicator. London: Longman.

Janoschka, Anja (2004): Web Advertising. New Forms of Communication on the Internet. Amsterdam: John Benjamins.

Katan, David (1999): Translating Cultures: An Introduction for Translators, Interpreters and Mediators. Manchester: St. Jerome.

KünZlI, Alexander (2001): Experts vs. Novices: La traduction du dialogue fictif dans les textes publicitaires. Babel. 47(4):323-342.

Lamizet, Bernard et Silem, Ahmed (1997): Dictionnaire encyclopédique des sciences de l'information et de la communication. Paris: Ellipses.

Léonard, Martine et Siguret, Françoise (1972): La route de l'expansion road ou l'impasse de la publicité bilingue. Meta. 17(1):56-71.

Li, Fengru et Shooshtari, Nader H. (2007): Multinational Corporations' Controversial Ad Campaigns in China - Lessons from Nike and Toyota. Advertising \& Society Review. 8(1):34-45.

MooIJ, Marieke de (2005): Global Marketing and Advertising: Understanding Cultural Paradoxes, Thousand Oaks: Sage.

O'BArr, William M. (2007): Advertising in China. Advertising and Society Review. 8(3):41-56.

PARÉ, Marcel (1972): Petit historique de la publicité française au Canada. Meta. 17(1):52-55.

Poyatos, Fernando, dir. (1997): Nonverbal Communication and Translation. Amsterdam: John Benjamins. 
430 Meta, LIV, 3, 2009

Quillard, Geneviève (1999) : Publicité, traduction et reproduction de la culture. Babel. 45(1):3952.

Quillard, Geneviève (2006): Translation and cultural mediation: The case of advertising in Canada. Translation and Interpreting Studies. 1(2):111-146.

SARtre, Jean-Paul (1940): L'imaginaire. Paris: Gallimard.

Sidiropoulou, Maria (2008): Cultural Encounters in Advertisement Translation. Journal of Modern Greek Studies. 26(2):337-362.

Sмітн, K. (2006): Rhetorical figures and the translation of advertising headlines. Language and Literature. 15(2):159-182.

Snell-Hornby, Mary, Jettmarová, Zuzana et Kaindl, Klaus, dir. (1995): Translation as Intercultural Communication. Selected papers from the EST Congress - Prague 1995. Amsterdam: John Benjamins.

Tatilon, Claude (1990): Le texte publicitaire: traduction ou adaptation? Meta. 35(1):243-250.

Tremblay, Gilles (1982): L’ABC du style publicitaire français. Montréal: Linguatech.

VANDAele, Sylvie et Lubin, Leslie (2005): Approche cognitive de la traduction dans les langues de spécialité: vers une systématisation de la description de la conceptualisation métaphorique. Meta. 50(2):415-431. 